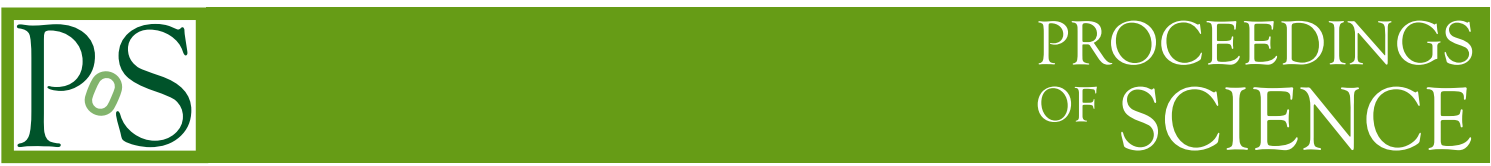

\title{
Hyperbolic PDEs with non-commutative time
}

\author{
Gandalf Lechner* \\ Institute for Theoretical Physics, University of Leipzig \\ E-mail: gandalf.lechnereuni-leipzig.de
}

In this talk, I will report on joint work with Rainer Verch [1] on hyperbolic PDEs with noncommutative time, i.e. linear integro-differential equations of the form $(D+\lambda W) f=0$, where $D$ is a (pre-)normal hyperbolic differential operator on $\mathbb{R}^{n}, \lambda \in \mathbb{C}$ is a coupling constant, and $W$ a regular integral operator which is non-local in time, so that a Hamiltonian formulation is not possible. Such equations appear in the context of wave or Dirac equations on non-commutative deformations of Minkowski space. It will be discussed that at small coupling, the hyperbolic character of $D$ is essentially preserved, unique advanced/retarded fundamental solutions can be constructed, and the acausal behavior of the solutions is well-controlled. Although the Cauchy problem is ill-posed in general, a scattering operator can be calculated which describes the effect of $W$ on the space of solutions of $D$.

It is also described how these results can be used for the analysis of classical and quantum field theories on non-commutative spaces.

References

[1] G. Lechner and R. Verch, Linear hyperbolic PDEs with non-commutative time, Preprint, arXiv:1307.1780

Frontiers of Fundamental Physics 14 - FFP14,

15-18 July 2014

Aix Marseille University (AMU) Saint-Charles Campus, Marseille

\footnotetext{
* Speaker.
} 